\title{
The Application Research of System Cooperative Design Engineering Based on MSFC Architecture
}

\author{
Hongjie Xu, Jinhu Ren, and Yingru Wang
}

\begin{abstract}
Many research institutions are exploring the best practice of model-based system engineering in order to improve the development quality of complex system, reduce the design cost and shorten the development cycle. The collaborative development of complex systems by MBSE is the focus of system engineering research and the foothold of engineering application. This paper proposes a new modeling idea-the MBSE system collaborative design idea based on MSFC data architecture, which used a unify data source and effective transfer of the model for system analysis and multi-professional collaborative design and used MSFC architecture to implement collaborative design for a certain aileron system to verified the feasibility of the theory.This paper verified the relevant requirements in the aileron system analysis process through the system modeling and simulation of the control subsystem, the hydraulic drive subsystem, the actuator subsystem and the rudder surface. The research shows that MSFC architecture was suitable for the collaborative design process of complex system that can be used as the modeling idea of MBSE engineering application.
\end{abstract}

Keywords MBSE - MSFC - System modeling - Collaborative development · Requirements closed-loop verification

H. Xu (凶)

Northwestern Polytechnica University, Xi' an 710000, China

e-mail: xuhj@avic-digital.com

H. Xu $\cdot$ J. Ren $\cdot$ Y. Wang

AVIC Digital Technology Co., Ltd., No. 7 Jingshun road, Chaoyang district, Beijing 100028, China 